Enfermagem Brasil 2018;17(1):10-7

\title{
ARTIGO ORIGINAL \\ Assistência de enfermagem nas complicações durante as sessões de hemodiálise
}

Eduardo Tavares Gomes, M.Sc. ${ }^{*}$, Maria José Silva dos Santos Nascimento**

${ }^{*}$ Enfermeiro, UPE/UEPB, ${ }^{*}$ Enfermeira, especialista em Enfermagem em Nefrologia pelo Centro de Capacitação Educacional, Faculdade Boa Viagem, Recife/PE

Recebido em 24 de junho de 2017; aceito em 3 de novembro de 2017.

Endereço de correspondência: Eduardo Tavares Gomes, Hospital das Clínicas da Universidade Federal Pernambuco, Avenida Prof Moraes Rego, 1235, Cidade Universitária, 50670-901 Recife PE, E-mail: edutgs@hotmail.com; Maria José Silva dos Santos Nascimento: mjssnascimento@hotmail.com

\section{Resumo}

Objetivo: Identificar as complicações e intervenções de enfermagem durante as sessões de hemodiálise. Métodos: Trata-se de um estudo descritivo de abordagem quantitativa, realizado em um serviço de hemodiálise. Resultados: Foram coletados 83 prontuários com registros de complicações durante sessões entre fevereiro e março de 2016. Foram registradas 149 complicações, sendo consideradas mais de uma possibilidade no período em estudo. As principais complicações apresentadas foram: pico hipertensivo $(25,50 \%)$, hipotensão $(24,83 \%)$, náusea $(18,12 \%)$, vômito $(10,07 \%)$ e cefaleia $(10,07 \%)$. Quanto ao registro das intervenções realizadas, foram encontrados 106 registros, e as que apresentaram maior registro foram: administrar reposição volêmica com soro fisiológico $(39,62 \%)$, administrar droga hipotensora (captopril), administrar reposição salínica (10,38\%). Conclusão: Recomenda-se a educação permanente da equipe de enfermagem para o entendimento clínico-fisiológico das intercorrências, reconhecimento precoce de sinais e sintomas envolvidos e ações validadas pela literatura para a correção ou minimização das complicações.

Palavras-chave: Enfermagem, nefrologia, diálise renal, assistência de enfermagem.

\section{Abstract \\ Nursing care in complications during hemodialysis sessions}

Objective: To identify the complications and nursing actions during hemodialysis sessions. Methods: It is a descriptive-quantitative study, carried out in a hemodialysis service. Results: We collected 83 records with records of complications during sessions between February and March 2016. We found 149 complications recorded, and considered more of one possibility in the study period. Major complications included the following: blood pressure peak $(25.50 \%)$, hypotension (24.83\%), nausea (18.12\%), vomiting (10.07\%) and headache $(10.07 \%)$. As for the recording of interventions, 106 records were found, and the most frequently recorded were: administer volume replacement with saline (39.62\%), administering hypotensive drug (captopril), administer saline replacement (10.38\%). Conclusion: We recommend continuing education for the nursing staff to the understanding of the clinical and physiological complications, early recognition of signs and symptoms involved and validated actions by the literature to correct or minimize complications.

Key-words: Nursing, nephrology, renal dialysis, nursing care.

\section{Resumen}

\section{Asistencia de enfermería en las complicaciones durante las sesiones de hemodiálisis}

Objetivo: Identificar las complicaciones e intervenciones de enfermería durante las sesiones de hemodiálisis. Métodos: Se trata de un estudio descriptivo de abordaje cuantitativo, realizado en un servicio de hemodiálisis. Resultados: Se recolectaron 83 prontuarios con registros de complicaciones durante sesiones entre febrero y marzo de 2016. Se registraron 149 complicaciones, siendo consideradas más de una posibilidad en el período en estudio. Las principales complicaciones presentadas fueron: pico hipertensivo $(25,50 \%)$, hipotensión $(24,83 \%)$, náusea $(18,12 \%)$, vómito $(10,07 \%)$ y cefalea $(10,07 \%)$. En cuanto al registro de las intervenciones realizadas, se encontraron 106 registros, y las que presentaron mayor registro fueron: administrar reposición volémica con suero fisiológico $(39,62 \%)$, administrar droga 
hipotensora (captopril), administrar reposición salínica (10,38\%). Conclusión: Se recomienda la educación permanente del equipo de enfermería para el entendimiento clínico-fisiológico de las intercurrencias, reconocimiento precoz de signos y síntomas involucrados y acciones validadas por la literatura para la corrección o minimización de las complicaciones.

Palabras-clave: Enfermería, nefrología, diálisis renal, asistencia de enfermería.

Introdução

A hemodiálise é o processo de filtragem e depuração do sangue de substâncias indesejáveis como a creatinina e a ureia que necessitam ser eliminadas da corrente sanguínea humana devido à deficiência no mecanismo de filtragem nos pacientes portadores de insuficiência renal crônica (IRC). Na hemodiálise, a transferência de solutos ocorre entre o sangue e a solução de diálise através de uma membrana por meio da difusão, que é o fluxo de soluto de acordo com o gradiente de concentração, sendo transferida massa de um local de maior concentração para um de menor concentração, a depender do peso molecular e características da membrana. A ultrafiltração é a remoção de líquidos através de um gradiente de pressão hidrostática e a convecção é a perda de solutos durante a ultrafiltração, quando ocorre o arraste de solutos na mesma direção do fluxo de líquidos através da membrana [1-3].

As complicações que ocorrem durante a sessão de hemodiálise podem ser eventuais, mas algumas são extremamente graves e fatais [1,4]. A principal complicação que ocorre durante a hemodiálise envolve as alterações hemodinâmicas decorrentes do processo de circulação extracorpórea e a remoção de um grande volume de líquidos em um espaço de tempo muito curto. As complicações mais comuns durante a hemodiálise são, em ordem decrescente de frequência, hipotensão $(20 \%-30 \%$ das diálises), cãibras $(5 \%-20 \%)$, náuseas e vômitos $(5 \%-15 \%)$, cefaleia $(5 \%)$, dor torácica (2\%-5\%), dor lombar (2\%-5\%), prurido $(5 \%)$, febre e calafrios $(<1 \%)$. As complicações menos comuns, mas sérias e que podem levar à morte incluem: a síndrome do desequilíbrio, reações de hipersensibilidade, arritmia, hemorragia intracraniana, convulsões, hemólise, embolia gasosa, hemorragia gastrintestinal, problemas metabólicos, convulsões, espasmos musculares, insônia, inquietação, demência, infecções, pneumotórax ou hemotórax, isquemia ou edema na mão e anemia [1,3-11].

Em um estudo de corte realizado em Alfenas-MG, verificou-se que $96,6 \%$ das complicações não ocorriam pela primeira vez, revelando alta reincidência e sugerindo a possibilidade de se prever e antecipar as intervenções [11]. A infecção de corrente sanguínea associada ao cateter central para hemodiálise é uma complicação que pode ser minimizada pela melhora da assistência de enfermagem e pela educação do paciente e que leva a perda do acesso venoso, internamento e sepse [12].

Ocorreu nos últimos anos um grande progresso em relação à segurança e a eficácia das máquinas de hemodiálise, tornando o tratamento mais seguro. Os equipamentos de hemodiálise contêm alarmes que indicam qualquer alteração que ocorra no sistema (detectores de bolhas, alteração de temperatura e do fluxo do sangue entre outros), mesmo assim, isso não garante que as complicações deixem de ocorrer [1,3-4]. Além disso, o paciente em tratamento renal crônico ainda estabelece uma relação de dependência desse equipamento, de uma equipe especializada, além da obrigatoriedade de aceitar e assumir um esquema terapêutico rigoroso para manutenção de sua vida [4]. A equipe de saúde treinada pode garantir um tratamento dialítico seguro, mesmo assim não há como se garantir a nãoocorrência de complicações, que no mais das vezes estão associadas às condições clínicas do paciente e a evolução da doença renal [4].

Além das complicações, ainda entram na conta dos potenciais riscos ao paciente os eventos adversos relacionados à terapia. Um estudo realizado com 25 profissionais de enfermagem revelou que todos relatam ter presenciado ou ter tido conhecimento de eventos adversos como cateter obstruído, retirada acidental da agulha da fístula e coagulação do sistema extracorpóreo. De todos os relatos de eventos adversos encontrados, apenas $42,8 \%$ estavam relacionados ao paciente, sendo o restante relacionado aos profissionais (falhas, despreparo, falta de atenção, falha na comunicação) e à organização do serviço (qualidade dos materiais, falta de recursos, sobrecarga de trabalho, recursos humanos inadequados, área física inadequada, ausência de protocolos específicos, etc. [13]).

A atuação da equipe de enfermagem diante destas complicações, desde a monitorização do paciente, a detecção de anormalidades e a rápida intervenção é essencial para a garantia de um procedimento seguro e eficiente para o paciente. A equipe de enfermagem tem importância muito grande na observação contínua dos pacientes durante a 
sessão, podendo ajudar a salvar muitas vidas e evitar muitas complicações ao fazer o diagnóstico precoce de tais intercorrências [3]. Pesquisas sobre a assistência de enfermagem, nesse cenário, possibilitam não só avaliar o serviço, como também verificar possibilidades de intervenção e de melhora.

O presente artigo teve por objetivo identificar as complicações e as intervenções de enfermagem durante as sessões de hemodiálise.

\section{Material e métodos}

Estudo descritivo, retrospectivo, de abordagem quantitativa, realizado no serviço de hemodiálise localizada na Zona da Mata de Pernambuco. A clínica atende 229 pacientes, divididos em seis turnos, com a cobertura garantida pelo Sistema Único de Saúde (SUS).

Foram coletados dados de 110 prontuários, dos quais 83 apresentaram registro de complicações durante as sessões. Foram considerados os registros dos meses de fevereiro e março de 2016, não se avaliando a reincidência ou a incidência de mais de uma complicação por paciente no em período anterior ao corte, mas contabilizando-se mais de um registro por período.

Os dados foram coletados dos prontuários, não incluídos aqueles cuja falta ou erros de preenchimento pudessem dificultar a análise. Para orientar a coleta, foi elaborado pelos pesquisadores um instrumento próprio contendo dados sociodemográficos, dados clínicos do momento da sessão, descrição da complicação e da intervenção referente.

Os dados foram analisados com recursos de epidemiologia descritiva (frequência absoluta, frequência relativa, médias, desvio-padrão, etc.) através do software estatístico SPSS 20.0.

A elaboração do projeto de pesquisa, bem como toda sua execução, foi pautada nos princípios bioéticos e nas orientações da Resolução do Conselho Nacional de Saúde ํo466/12. A etapa de coleta de dados se deu apenas após a anuência da clínica, a apreciação e aprovação do Comitê de Ética em Pesquisa do Hospital Otávio de Freitas - CAAE: 04137012.4.0000.5200.

\section{Resultados}

Os pacientes avaliados foram predominantemente do sexo masculino $(66,7 \%)$, com menos de 60 anos $(67,61 \%)$, sendo a idade média de $51,77 \pm 15,07$, a maioria sem vínculo formal $(49,4 \%)$ ou aposentado (42,17\%), com renda de 4 a 6 salários mínimos $(62,65 \%)$, com escolaridade de até 10 anos (74,694\%), apesar de 12,05\% serem analfabetos (Tabela I).

O principal acesso venoso utilizado foi a fístula arterio-venosa $(69,88 \%)$, seguido pelo acesso venoso central com cateter duplo lúmen $(30,12 \%)$. A duração média das sessões foi de $3,9 \pm 0,27$ horas (eram planejadas para 4 horas, mas em 8 foram encerradas antecipadamente em virtude das complicações apresentadas, todas na última hora da sessão).

A totalidade dos registros das complicações foi realizada por técnicos de enfermagem. Foram registradas 149 complicações, sendo consideradas mais de uma possibilidade no período em estudo. As principais complicações apresentadas foram: pico hipertensivo (25,50\%), hipotensão $(24,83 \%)$, náusea $(18,12 \%)$, vômito $(10,07 \%)$ e cefaleia $(10,07 \%)$. As complicações calafrio $(1,34 \%)$ e hipertermia $(0,67 \%)$ foram as que tiveram menor registro. (tabela II).

Quanto ao registro das intervenções realizadas, foram encontrados 106 registros, ou seja, das 149 complicações, 43 registros não se apresentaram juntos aos registros das respectivas intervenções $(29,45 \%)$. As intervenções que apresentaram maior registro foram: administrar reposição volêmica com soro fisiológico $(39,62 \%)$, administrar droga hipotensora (captopril), administrar reposição salínica $(10,38 \%)$. As intervenções com menor registro foram: aquecer o paciente com manta (1,89\%), administrar antitérmico (não-especificado) $(0,94 \%)$ (tabela III). 
Tabela I - Caracterização dos pacientes quanto ao sexo, idade, vínculo empregatício, escolaridade e renda ( $N=83$ ). Recife/PE, 2017.

\begin{tabular}{lll}
\hline Variáveis & $\mathbf{N}$ & $\%$ \\
\hline Sexo & 55 & 66,27 \\
Masculino & 28 & 33,73 \\
Feminino & & \\
Idade & 23 & 26,71 \\
Até 40 anos & 15 & 18,07 \\
De 41 a 50 anos & 19 & 22,89 \\
De 51 a 60 anos & 16 & 19,28 \\
De 61 a 70 anos & 10 & 12,05 \\
Mais que 70 anos & & \\
Vínculo empregatício & 1 & 1,2 \\
Com vínculo formal & 41 & 49,4 \\
Sem vínculo formal & 6 & 7,23 \\
Desempregado & 35 & 42,17 \\
Aposentado & & \\
Renda & 28 & 33,73 \\
Até 3 salários & 52 & 62,65 \\
De 4 a 6 salários & 3 & 3,61 \\
Sem registro & & \\
Escolaridade & 10 & 12,05 \\
Analfabeto & 30 & 36,14 \\
Até 4 anos & 32 & 38,55 \\
De 5 a 10 anos & 10 & 12,05 \\
De 10 a 14 anos & 1 & 1,2 \\
Mais de 15 anos & & \\
\hline
\end{tabular}

Tabela II - Distribuição das complicações apresentadas por pacientes durante sessão de hemodiálise (N=149). Recife/PE, 2017.

\begin{tabular}{lll}
\hline Complicações & $\mathbf{n}$ & $\%$ \\
\hline Pico hipertensivo & 38 & 25,50 \\
Hipotensão & 37 & 24,83 \\
Náusea & 27 & 18,12 \\
Vômito & 15 & 10,07 \\
Cefaleia & 15 & 10,07 \\
Câimbra & 14 & 9,40 \\
Calafrio & 2 & 1,34 \\
Hipertermia & 1 & 0,67 \\
- registros múltiplos & & \\
\hline
\end{tabular}

Tabela III - Registro das intervenções perante as complicações apresentadas durante sessão de hemodiálise. Recife/PE, 2017.

\begin{tabular}{lll}
\hline Intervenções & $\mathbf{n}$ & $\%$ \\
\hline Administrar reposição volêmica com soro fisiológico & 42 & 39,62 \\
Administrar droga hipotensora - captopril & 30 & 28,30 \\
Administrar reposição salínica & 11 & 10,38 \\
Administrar droga hipotensora - atensina & 6 & 5,66 \\
Administrar analgésico - dipirona & 5 & 4,72 \\
Administrar antiemético & 4 & 3,77 \\
Administrar reposição volêmica com soro glicosado & 3 & 2,83 \\
Administrar droga hipotensora - atensina + captopril & 2 & 1,89 \\
Aquecer o paciente com manta & 2 & 1,89 \\
Administrar antitérmico & 1 & 0,94 \\
\hline
\end{tabular}

\section{Discussão}

O perfil da amostra corrobora o de outros estudos no tocante a gênero, idade, escolaridade [11,14-7]. Em estudo recente realizado no intuito de verificar a associação entre 
os modos de enfrentamento e as variáveis sociodemográficas de pessoas em hemodiálise, ficou demonstrado menores escores relacionados ao enfrentamento entre os homens e que os idosos apresentavam maiores escores de enfrentamento focados na emoção (confronto, afastamento, fuga-esquiva). O mesmo estudo encontrou que pessoas com escolaridade $\geq 12$ anos tiveram escores médios mais elevados para o enfrentamento focado no problema, em relação às pessoas com menor escolaridade, assim como menores escores de enfrentamento para o subgrupo de menor renda ou que não trabalhava [15].

Outros autores refletiram sobre o convívio com a doença renal crônica e a hemodiálise, concluindo que a experiência promove uma ruptura biográfica na vida do indivíduo e que o mesmo passa por sentimentos ambíguos em sua relação com a máquina de hemodiálise e mudanças na qualidade de vida, assim como com a equipe que o assiste [17-8].

A fístula arterio-venosa $(69,88 \%)$ foi predominante no estudo, assim como já foi observado que é a escolha dentre os acessos vasculares no país [18]. A fístula confeccionada para diálise está relacionada a eventos adversos como a retirada acidental da agulha, sangramento, etc., mas não a complicações durante a sessão [12-13].

Os técnicos de enfermagem foram responsáveis pela totalidade dos registros das intercorrências em estudo. Isso pode estar relacionado ao dimensionamento de pessoal e a rotina de registro por parte dos técnicos de cada etapa da sessão de hemodiálise. Não foi possível verificar qual o profissional que reconheceu os sinais e sintomas relacionados, apenas o responsável pelo registro em prontuário. As unidades que prestam serviço de hemodiálise devem ter bem elaborados e revisados os instrumentos que permitam o registro da sistematização da assistência de enfermagem e não apenas o registro de dados da sessão [20]. Em $29,45 \%$ das complicações não houve simultaneamente o registro das intervenções, demonstrando uma fragilidade do processo. Não foi possível avaliar ainda se os $27(24,54 \%)$ prontuários estudados que não compuseram a amostra tiveram registro de complicações porque as mesmas não ocorreram ou se por outro motivo não foi realizado o registro.

O enfermeiro deve ser responsável pela sistematização além da rotina de registro de dados como peso, temperatura e pressão arterial realizados pelos técnicos, visto que apenas pelo processo de enfermagem poderá o enfermeiro realizar intervenções que abarquem dimensões subjetivas do paciente, muitas vezes negligenciadas [15,20-1]. Outra pesquisa mostrou que o uso de check-list para registro da sessão contendo dados pré-sessão, do início e do encerramento, aumenta a qualidade e a segurança durante o tratamento [22].

A hipotensão arterial foi observada em $24,83 \%$ dos pacientes. É uma complicação relacionada à taxa de ultrafiltração, temperatura do dialisato, redução do volume líquido circulante, perda de sódio, redução do débito cardíaco. É um reflexo primário da retirada excessiva de líquidos durante a sessão. Deve ser investigada ainda a perda de sangue nas conexões e ruptura das membranas, ou sangramento interno. Como intervenção associada, a mais utilizada foi a reposição volêmica, não sendo citadas a mudança para a posição de Trendelemburg ou administração de oxigênio por via nasal $[4,11-12,15]$. Há referências que indicam que temperaturas mais baixas do dialisato pode prevenir a hipotensão por causar vasoconstrição periférica e que deve ser retirado mais líquido na primeira hora da sessão, reduzindo a filtração nas horas subsequentes [23].

O pico hipertensivo foi evidenciado em $25,5 \%$ dos pacientes. Está associada na literatura à ativação do sistema renina-angiotensina secundária a depleção de volume, à sobrecarga de volume, ansiedade ou síndrome de desequilíbrio 4,11-12,15,24. O tratamento é feito pela correção da causa e pela administração de hipotensores, como nifedipina e captopril. Nas situações observadas através do prontuário, a nifedipina não foi utilizada, em substituição pela atensina. No caso de ansiedade, a psicoterapia e os sedativos prestam boa ajuda, bem como a confiança desenvolvida na equipe de enfermagem [12,15].

Nauseia e vômitos apresentaram-se, respectivamente, em 18,12\% e $10,07 \%$ dos casos. Geralmente ocorrem secundárias às alterações pressóricas, síndrome do desequilíbrio, ansiedade, ingestão de alimentos durante a sessão e aumento do cálcio sérico. Sugere-se a administração de antieméticos, correção da pressão arterial e diminuição da velocidade do fluxo sanguíneo com prolongamento proporcional da sessão [4,11-12,15,24]. A intervenção principal encontrada na amostra foi a correção dos níveis pressóricos, sendo os antieméticos registrado em apenas $3,77 \%$ dos casos.

A cefaleia foi registrada em $10,07 \%$ dos casos. É um sintoma frequentemente associado à hemodiálise, sendo indicadas como causas: a hipertensão arterial, hipotensão arterial, alterações no peso corporal, ansiedade, síndrome do desequilíbrio, uso de solução de diálise contendo acetato, abstinência de cafeína (uma vez que a sua concentração sanguínea é 
reduzida agudamente durante a HD). Assim como para as náuseas e vômitos, uma redução na velocidade de fluxo sanguíneo durante a parte inicial da diálise pode ser tentada. O uso de analgésicos via oral ou parenteral deve ser associado à supressão da causa e foi registrado em $4,72 \%$ dos prontuários, sendo a dipirona a droga escolhida.

Apesar de não ter sido registrada, a hipoglicemia é um dos riscos de complicações durante as sessões de diálise. Investigando-se a resposta glicêmica de pacientes com diabetes tipo 2, um estudo recente revelou que as soluções de diálise sem ou pobres em glicose $(55 \mathrm{md} / \mathrm{dl})$ apresentaram maior risco de hipoglicemia [25].

Também não foram relatados casos de parada cardiorrespiratória. Um estudo americano demonstrou que o risco de parada cardíaca no período peri-dialítico é maior entre pacientes com doença coronariana, insuficiência cardíaca, níveis baixos de potássio no dialisato, elevado cálcio sérico, hipoalbuminemia, baixo peso, baixa hemoglobina [26].

As complicações registradas foram condizentes com a literatura atual, bem como as intervenções de enfermagem [27]. Um estudo nacional divulgou que as principais intervenções de enfermagem descritas na literatura são: monitoramento hidroeletrolítico, verificação de sinais vitais, administração de medicamentos e orientações ao paciente [27]. Contudo, nos registros avaliados não foram consideradas as atividades de monitoramento e de orientação aos pacientes como específicas para as complicações nas sessões.

Cabe ainda, uma reflexão importante sobre o uso de taxonomias para o registro da Sistematização da Assistência de Enfermagem na hemodiálise. Pesquisas acerca da temática verificaram que os diagnósticos de enfermagem mais relevantes para a prática clínica em hemodiálise são: risco de infecção, risco de sangramento, risco de quedas, risco de choque, risco de resposta alérgica, volume de líquido excessivo, risco de desequilíbrio do volume de líquidos, risco de glicemia instável, risco de desequilíbrio eletrolítico, nutrição desequilibrada: menos do que as necessidades corporais, risco de perfusão renal ineficaz, risco de perfusão tissular cardíaca, diminuída, dor crônica, náusea, dor aguda, comportamento de saúde propenso a risco, controle familiar ineficaz do regime terapêutico [28]. Destaca-se que nos impressos para esse registro de assistência, através destes diagnósticos elencados e outros mais disponíveis, é possível descrever ou reconhecer as complicações durante a assistência. A taxonomia deve ser de uso de todos os profissionais, pois comunica o diagnóstico de enfermagem além de propiciar o registro de intervenções realizadas [29-31].

\section{Conclusão}

É de responsabilidade legal do enfermeiro a supervisão do serviço de hemodiálise, bem como a assistência prestada aos pacientes gravemente enfermos, compreendendo as situações de emergência. Sendo assim, ele é o profissional responsável por avaliar os sinais e sintomas dos pacientes, conferir as prescrições médicas relativas, prescrever as intervenções de enfermagem, orientá-las e supervisioná-las.

Apesar de o estudo ter por limitações o pequeno número de pacientes e retratar a realidade de apenas um centro de diálises, o mesmo serve para a reflexão sobre a atuação da equipe de enfermagem nas situações delicadas que podem ocorrer durante as sessões de hemodiálise.

Reforçamos, por fim, após a análise dos resultados sob a luz da literatura, a necessidade da revisão dos fluxos assistenciais de forma a garantir que o enfermeiro tome lugar nas complicações, revisão dos impressos utilizados para a sistematização da assistência de enfermagem; educação permanente da equipe de enfermagem para o entendimento clínicofisiológico das intercorrências, reconhecimento precoce de sinais e sintomas envolvidos e ações validadas pela literatura para a correção ou minimização das complicações; e elaboração de um roteiro sistematizado para a educação do paciente na primeira consulta de forma a ajudá-lo a compreender as alterações que seu corpo e sua rotina sofrerão ao longo da hemodiálise.

1. Riella MC. Princípios de nefrologia e distúrbios hidroeletrolíticos. Rio de Janeiro: Guanabara Koogan; 2010.

2. Daugirdas JT, Blake PG, Ing TS. Manual de diálise. Rio de Janeiro: Medsi; 2008.

3. Fermi MRV. Manual de diálise para enfermagem. $2^{\underline{a}}$ ed. Rio de Janeiro: Medsi; 2010. 
4. Castro MCM. Atualização em diálise: complicações agudas em hemodiálise. J Bras Nefrol 2001;23(2):108-13.

5. Calixto RC, Lorençon M, Corrêa MSMF, Cruz AP, Martins LC, Barretti P et al. Intercorrências dialíticas em hemodiálise. J Bras Nefro 2003;25(Supl1):7.

6. Reis EMK, Silva TD, Garcia TSE. Eventos mórbidos intradialíticos: tipos e frequência em relação ao dialisato com e sem glicose a 1\%. J Bras Nefro 2003;25(Supl1):7; HD ENF 17.

7. Reis EMK, Silva TS. Eventos mórbidos intradialíticos em nefropatas diabéticos e não diabéticos: caracterização e condutas. J Bras Nefro 2003;25(Supl1):7; HD ENF 18.

8. Dias TS, Reis EMK. Eventos mórbidos em sessões de hemodialíticas: otimização das ações de enfermagem. J Bras Nefro 2003;25(Supl 1):9; HD ENF 24.

9. Rodrigues MCS. A atuação do enfermeiro no cuidado ao portador de insuficiência renal crônica no contexto biotecnológico da hemodiálise. Nursing 2005;82(8):135-42.

10. Oliveira FC, Alves MDS, Bezerra AP. Co-morbidades e mortalidade de pacientes com doença renal: atendimento terceirizado de nefrologia. Acta Paul Enferm 2009;22:47680.

11. Terra FS, Costa AMDD, Figueiredo ET, Morais AM, Costa MD, Costa RD. As principais complicações apresentadas pelos pacientes renais crônicos durante as sessões de hemodiálise. Rev Bras Clin Med 2010;8(3):187-92.

12. Nascimento $C D$, Marques IR. Intervenções de enfermagem nas complicações mais frequentes durante a sessão de hemodiálise: revisão da literatura. Rev Bras Enferm 2005;58(6):719-22.

13. Sousa MRG, Silva AEBC, Bezerra ALQ, Freitas JS, Miasso Al. Eventos adversos em hemodiálise: relatos de profissionais de enfermagem. Rev Esc Enferm USP 2013;47(1):76-83.

14. Bertolin DC, Pace AE, Kusumota L, Haas V. Associação entre os modos de enfrentamento e as variáveis sociodemográficas de pessoas em hemodiálise crônica. Rev Esc Enferm USP 2011;45(5):1070-6.

15. Dallé J, Lucena AF. Diagnósticos de enfermagem identificados em pacientes hospitalizados durante sessões de hemodiálise. Acta Paul Enferm 2012;25(4):504-10.

16. Xaveir BLS, Santos I, Almeida RF, Clos AC, Santos MT. Individual and clinical characteristics of clients with chronic kidney disease replacement therapy. Rev Enferm UERJ 2014;22(3):314-20.

17. Abreu IS, Santos $C B$. Qualidade de vida relacionada à saúde de pacientes em hemodiálise. Rev Enferm UERJ 2013;21(1):95-00.

18. Mattos M, Maruyama SAT. A experiência de uma pessoa com doença renal crônica em hemodiálise. Rev Gaúcha Enferm 2010;31(3):428-34.

19. Linardi F, Linardi FF, Bevilacqua JL, Morad JFM, Costa JA, Miranda Júnior F. Acesso vascular para hemodiálise: avaliação do tipo e local anatômico em 23 unidades de diálise distribuídas em sete estados brasileiros. Rev Col Bras Cir 2003;30(3):183-93.

20. Oliveira SM, Ribeiro RCHM, Ribeiro DF, Lima LCEQ, Pinto MH, Poletti NAA. Elaboração de um instrumento da assistência de enfermagem na unidade de hemodiálise. Acta Paul Enferm 2008;21:169-73.

21. Lata AGB, Albuquerque JG, Carvalho LASBP, Lira ALBC. Diagnósticos de enfermagem em adultos jovens em tratamento de hemodiálise. Acta Paul Enferm 2008;21(Número Especial):160-3.

22. Marcelli D, Matos A, Sousa F, Peralta R, Fazendeiro J, Porra A et al. Implementation of a quality and safety checklist for haemodialysis sessions. Clin Kidney J 2015;8(3):26570.

23. Agarwal R. How can we prevent intradialytic hypotension? Curr Opin Nephrol Hypertens 2012;21(6):593-9.

24. Veiga HC, Pinheiro LA, Lugon JR. Revisão/atualização em diálise: alterações cardiovasculares em pacientes em hemodiálise regular. J Bras Nefrol 2004;20(3):33641.

25. Burmeister JE, Miltersteiner DR, Burmeister BO, Campos JF. Risk of hypoglycemia during hemodialysis in diabetic patients is related to lower pre-dialysis glycemia. Arch Endocrinol Metab 2015;59(2):137-40.

26. Flythe JE, Li NC, Lin SF, Brunelli SM, Hymes J, Lacson E Jr. Associates of cardiopulmonary arrest in the perihemodialytic period. Int J Nephrol 2014;2014:961978. 
27. Costa RHS, Dantas ALM, Leite EMD. Complicações em pacientes renais durante sessões hemodialíticas e intervenções de enfermagem. Rev Pesqui Cuid Fundam online 2015;7(1):2137-46.

28. Lemes MMD, Bachion MM. Enfermeiros atuantes em hemodiálise indicam diagnósticos de enfermagem relevantes na prática clínica. Acta Paul Enferm 2016;29(2):185-90.

29. Stumm EM, Fernandes KRM, Guido LA, Benetti ERR, Belasco AGS, Sesso RCC et al. Intervenção educacional de enfermagem para redução da hiperfosfatemia em pacientes em hemodiálise. Rev Bras Enferm 2017;70(1):31-8.

30. Debone MC, Pedruncci ESN, Candido MCP, Marques S, Kusumota L. Diagnósticos de enfermagem em idosos com doença renal crônica em hemodiálise. Rev Bras Enferm 2017;70(4):800-5.

31. Poveda VB, Alves JS, Santos EF, Garcia AEM. Diagnósticos de enfermagem em pacientes submetidos à hemodiálise. Enferm Global 2014;(34):70-81. 